\title{
Theoretical Study of the Transesterification of Triglycerides to Biodiesel Fuel under Various Conditions*
}

\author{
Y. Asakuma** ${ }^{1}$, O. Kawanami ${ }^{1}$, K. Maeda ${ }^{1}$, H. Kuramochi ${ }^{2}$ and K. Fukui ${ }^{1}$ \\ ${ }^{1}$ University of Hyogo, Himeji, Japan \\ ${ }^{2}$ National Institute of Environmental Study, Tsukuba, Japan \\ E-mail: asakuma@eng.u-hyogo.ac.jp
}

\begin{abstract}
The transesterification of triglycerides under various conditions was considered in terms of the activation energy obtained from molecular orbital calculations. The transesterification reaction proceeds via a cyclic transition state, consisting of the carboxyl carbon and the alcohol. Moreover, the reaction pathway was shown by an activation energy analysis and an electrostatic potential distribution.
\end{abstract}

\section{Keywords: Biodiesel fuel; transesterification reaction; electrostatic potential.}

\section{Introduction}

Biodiesel is becoming increasingly important as an alternative fuel for diesel engines because of diminishing petroleum reserves and as part of the effort to protect the global environment. Biodiesel is made from renewable biological sources such as vegetable oils and animal fats which consist of the simple alkyl esters of fatty acids, and is considered to contribute much less to global warming than fossil fuels (Demirbas, 2004).

Transesterification is the reaction of a triglyceride such as vegetable oil with an alcohol, usually methanol, in the presence of an acidic or alkaline catalyst to produce fatty acid esters and glycerol. A base-catalyzed transesterification process is normally adopted for biodiesel production because alkaline metal alkoxides such as sodium methoxide and hydroxides are more effective than acid catalysts. In addition to this, reaction rates under acid catalysis are slower than those under alkaline conditions (Sharma et al., 2008). Moreover, biodiesel fuel can also be prepared from fatty acids in subcritical and supercritical methanol or water under catalyst-free conditions (Minami et al., 2006; Alenezi et al., 2009). In this case, high energy is required for hydrolysis because of the low reactivity of water.

A number of researchers have demonstrated the importance of variables such as reaction rate and selectivity of the catalyst in transesterification. Generally, the properties of biodiesel fuel are strongly influenced by both the structure and concentration of the fatty acid esters, which depend on the feedstock oil, such as palm, soybean corn or sunflower, and the reaction conditions such as temperature and catalyst. We have previously studied the solubility properties of a mixture of triglycerides and methanol in a vapor-liquid-liquid three-phase equilibrium to improve the reaction rate (Maeda et al., 2008) and also analyzed the decomposition mechanism using molecular orbital calculations (Asakuma et al., 2009).

A typical transesterification of a triglyceride ester bond is shown in Fig. 1(a) and 1(b). It consists of consecutive reversible reactions under acidic or basic conditions. The triglyceride is converted stepwise to a diglyceride, a monoglyceride and finally, to glycerol by removal of an alkyl in each step, as shown in Fig. 2, where $\mathrm{R}^{1}, \mathrm{R}^{2}$ and $\mathrm{R}^{3}$ represent long chain alkyl groups (Boocock et al., 1998; Jin F. et al., 2007; Demirbas, 2008; Barakos et al., 2008). Accordingly, we expect that systematic theoretical estimations of transesterification reaction activation energies are essential to clarify the transesterification pathways and the complex acid or base-catalyzed transesterification mechanism. This study therefore focuses on the mechanisms of transesterification and hydrolysis, using molecular orbital calculations to analyze the mechanism. Various types of behavior were considered in terms of the activation energy and electrostatic potential (ESP) distribution obtained from molecular orbital calculations. These types of behavior include the effect of hydrolysis and transesterification reaction mechanisms under acidic or basic conditions on reactivity as well as the pathway from triglyceride to diglyceride and monoglyceride.

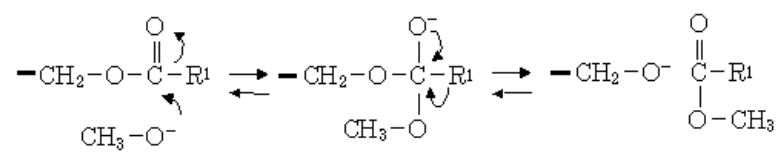

(a)

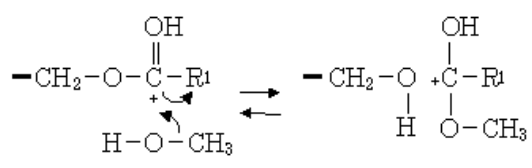

(b)

Figure 1. Triglyceride transesterification mechanism: (a) basic conditions and (b) acidic conditions. 


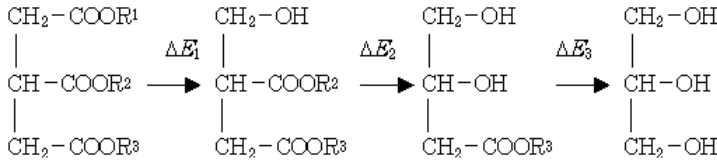

(a)

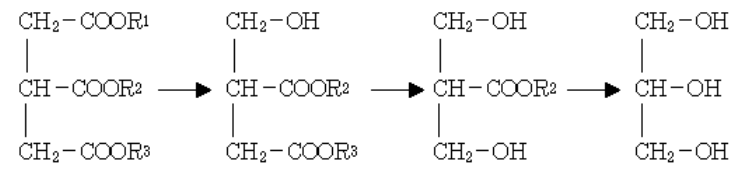

b)

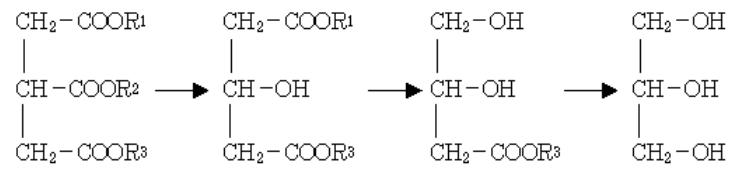

c)

Figure 2. Three different pathways of transesterification: a) $1 \rightarrow 2 \rightarrow 3$, b) $1 \rightarrow 3 \rightarrow 2$ and (c) $2 \rightarrow 1 \rightarrow 3$.

\section{Calculations}

Figure 1 shows the transesterification reaction of a triglyceride such as vegetable oil or animal fat. The numbers 1, 2 and 3 in Fig. 2 indicate the position of the carboxyl group, either in the center (2) or on the outside (1 and 3). The triglyceride is split into its components via transesterification, catalyzed by the addition of an acid or a base. Base-catalyzed transesterification is the process of exchanging the alkoxy group of an ester compound for another alkoxy group of a different alcohol such as methanol as shown in Fig. 1(a). The fatty acid monoalkyl ester can be used as biodiesel fuel in diesel engines. After the ester linkage of triglyceride is broken by an alkoxy group under basic conditions, three fatty acid esters and glycerol (glycerin) are produced via a transesterification reaction. When the carbonyl is attacked by the alkoxy group, the reaction proceeds through a tetrahedral intermediate or transition state (Hori et al., 1999). Transesterification with an acid catalyst is similar, as shown in Fig. 1(b). However, in the hydrolysis reaction, the methoxide anion and methanol are replaced by a hydroxide anion and water, respectively. The characteristics of these reactions are best considered by evaluation of the activation energy.

It is first necessary to calculate the optimized size of the ground state and transition state for the transesterification, because each state has a different energy. In this study, ab initio molecular orbital calculations were chosen because a semi-empirical calculation (Neyda et al., 2008) might be insufficient for the evaluation of the electronic structure. The quantum data of each state is derived using Gaussian software (Gaussian R 03W Ver. 6, Gaussian, Inc.) (Hori et al., 1999). The structures of ground state molecules (triglyceride, alkoxy group and fatty acid ester) and the transition state of the transesterification reaction were simulated. The activation energy of the transesterification reaction was obtained from optimized structures calculated under minimum energy configurations from the initial approximate coordinates of the atoms. The basis set used in this calculation was HF/STO-3G (Asakuma et al., 2009).

Transesterification and hydrolysis reactions under acidic and basic conditions were considered. In this case, methanol and water were used as the reactants for transesterification and hydrolysis, respectively. Finally, the main reaction pathway was predicted. The triglyceride has three ester bonds which are transesterified one by one as shown in Fig. 2. The activation energies obtained from these calculations show which ester bonds are easier to transesterify initially, and show the main pathways.

\section{Results and Discussion}

Every transesterification and hydrolysis proceeds via the following transition state. The oxygen atom of the reactant attacks the carbon of the carboxyl group, and a four or fivemembered cyclic transition state with a tetrahedral intermediate carbon and an alkoxy group is formed (Hori et al., 1999). Transesterification with an alkoxy group under basic conditions is shown in Fig. 3(a); the cyclic transition state is five-membered (Asakuma et al., 2009). In this study, the mechanism under acidic conditions is shown as follows. First, the carbonyl oxygen is protonated, then methanol attacks the carboxyl carbon, and a five-membered cyclic transition state is formed simultaneously as shown in Fig. 3(b) (Sharma et al., 2008).

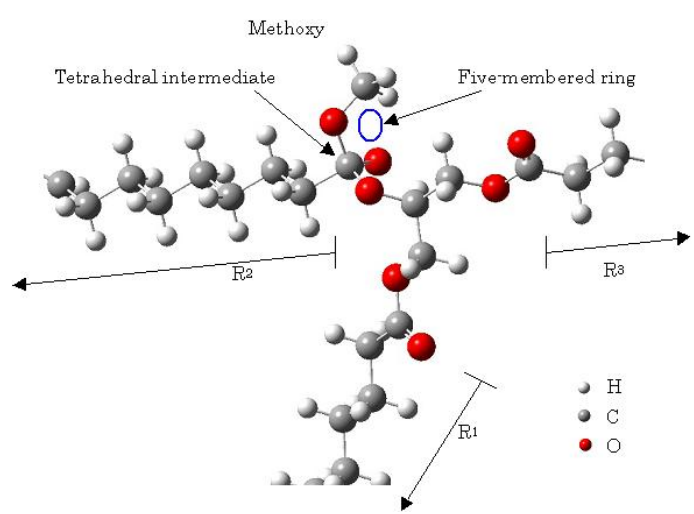

(a)

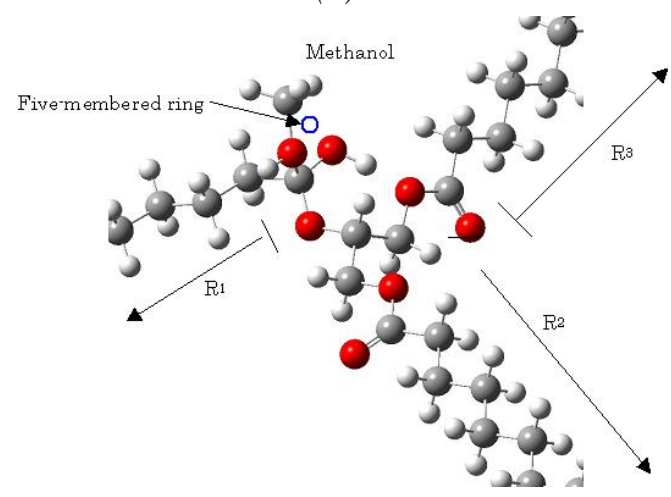

(b)

Figure 3. Cyclic transition states involving methanol during transesterification under: a) basic conditions and b) acidic conditions.

Figures 4(a) and 4(b) show the electrostatic potential (ESP) distribution around the ester bond of the triglyceride. Electrons of the carboxyl carbon are pulled toward the neighboring oxygen atoms because of the higher electronegativity of oxygen. The ESP distribution shows higher values under acidic conditions than under basic conditions because the carbonyl oxygen is protonated, as shown in Fig. 1(b). ESP values of the carboxyl carbon become highest in the triglyceride molecule. In the same way, ESP near the oxygen atoms shows the lowest value, and thus the ESP values of oxygen and the carboxyl carbon 
of the triglyceride become negative and positive, respectively. Similarly, negative ESP values are concentrated around the oxygen atom of the methoxide anion and methanol oxygen as shown in Figs. 4(c) and 4(d). Accordingly, transition states are formed by two forces: the electrostatic force between the oxygen atom of the methanol and the carboxyl carbon, and the hydrogen bond between the hydrogen atom of the methanol and the carbonyl oxygen. The cyclic transition state appeared in every calculation of the optimal structure, and this tetrahedral structure is therefore defined as a transition state. The importance of the cyclic transition state has been reported for other transesterification reactions (Hori et al., 1999).

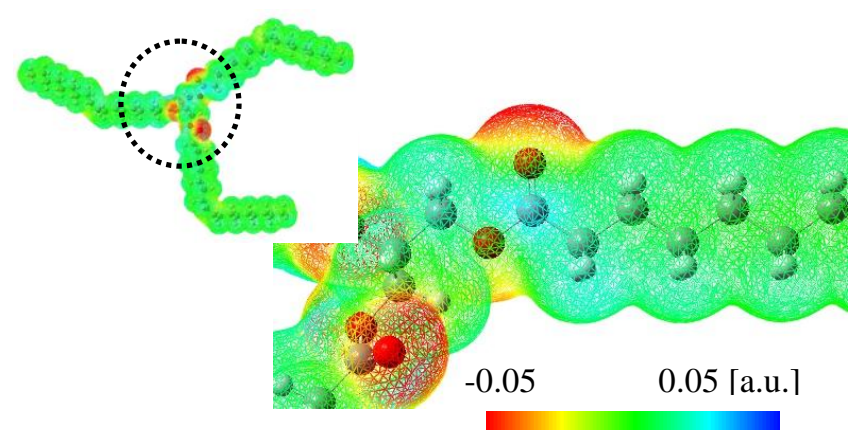

(a)

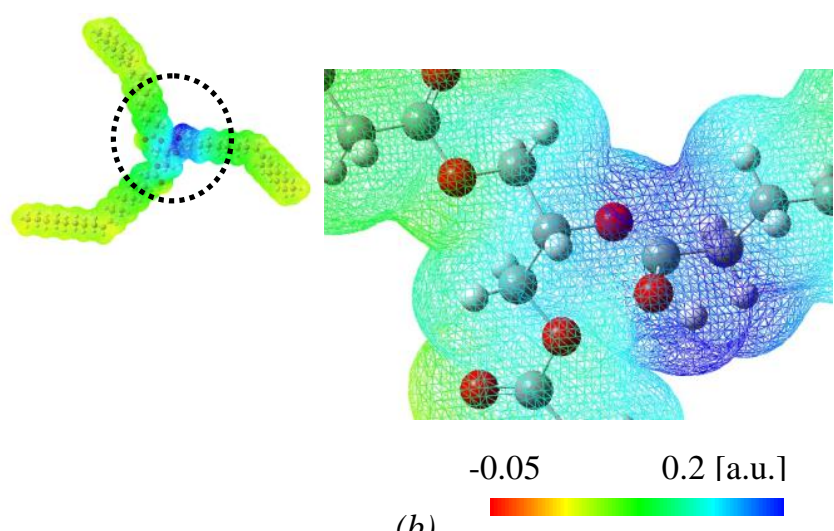

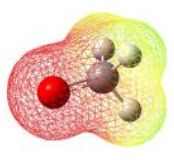

(c)

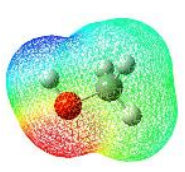

(d)
$-0.05$

0.05 「а.u.
Figure 4. Electrostatic potential distribution:

a) triglyceride under basic conditions,

b) triglyceride under acidic conditions,

c) methoxide anion and d) methanol.

\subsection{Pathway of transesterification and hydrolysis under basic conditions}

Figure 5 shows the activation energy of each of the three stages of transesterification and hydrolysis. According to the activation energy values, pathway (c) is favored because the total activation energy of pathway (c) is lower than the others. This means that transesterification and hydrolysis of a center ester bond is performed in preference to outside ester bonds. Moreover, transesterification reactions of diglycerides and monoglycerides $\left(\Delta E_{2}\right.$ and
$\Delta E_{3}$ ), which have larger activation energies, are more difficult than those of triglycerides $\left(\Delta E_{1}\right)$. This behavior corresponds to experimental data wherein transesterification is incomplete, and a small portion of monoglyceride and diglyceride remains (Boocock et al., 1998; Barakos et al., 2008). However, hydrolysis shows a higher activation energy than transesterification by the methoxide anion. This is a result of the ring size of the transition state; in other words, the four-membered cyclic transition state during hydrolysis is more unstable than the five-membered cyclic transition state of transesterification. This behavior is consistent with the high energy and harsh conditions that are required for the hydrolysis reaction to take place (Minami et al., 2006; Alenezi et al., 2009).

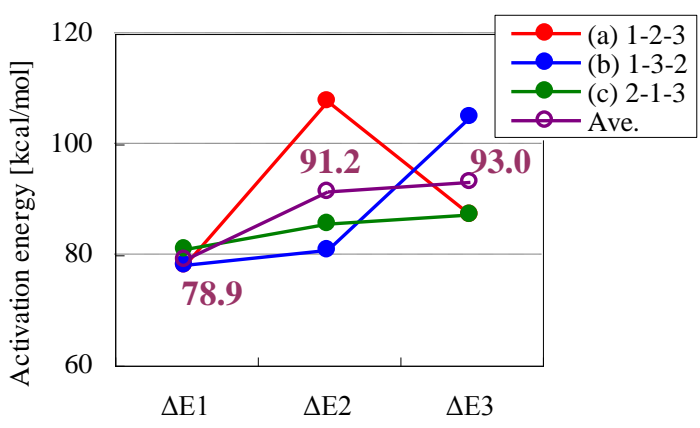

(a)

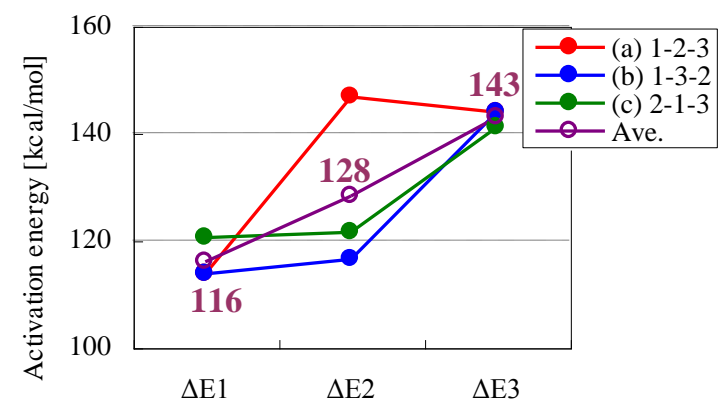

(b)

Figure 5. Activation energy of the transesterification pathway under basic conditions:

a) transesterification and b) hydrolysis.

\subsection{Pathway of transesterification and hydrolysis under acidic conditions}

The activation energy of each of the three stages of hydrolysis and transesterification under acidic conditions is shown in Fig. 6, and the same trend was observed under basic conditions. The activation energy under acidic conditions shows lower values than those of basic conditions although the acid-catalyzed reaction is slower in general (Sharma et al., 2008). This can be explained by the optimized structure of the transition state. In the case of acid-catalyzed transesterification and hydrolysis, a sevenmembered cyclic transition state is formed by intramolecular bonds between the center and side carboxyl bonds as shown in Fig. 7. This transition state includes the ester linkage, which is broken by methanol or a hydroxide ion during the transesterification reaction. The formation of the larger cyclic transition state makes the final step in transesterification and hydrolysis less favorable. This stable structure is formed when the center carbonyl bond is 
attacked by a methanol or a water molecule. Moreover, the geometrical position of the reactant affects the formation of a larger cyclic transition state.

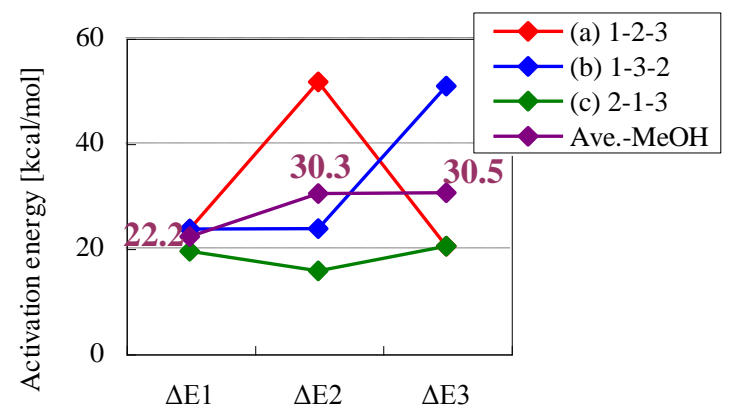

(a)

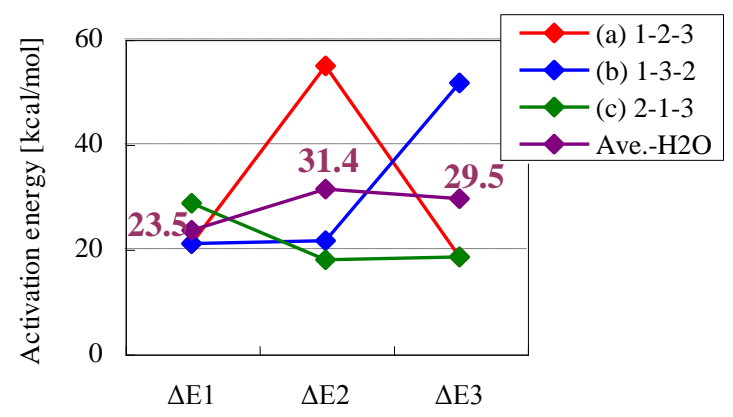

(b)

Figure 6. Activation energy of the transesterification pathway under acidic conditions:

a) transesterification and b) hydrolysis.

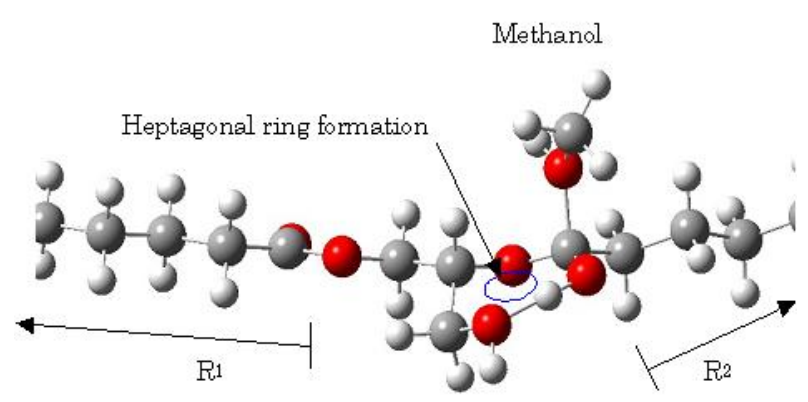

Figure 7. Seven-membered cyclic transition state during transesterification.

\section{Conclusions}

The activation energies of triglyceride transesterification and hydrolysis reactions under acidic and basic conditions were calculated by using Gaussian software to clarify the mechanism. The values show a difference in reactivity for transesterification and hydrolysis. Formation of the fatty acid alkyl ester by the addition of an alkoxy group was considered in terms of the activation energy. Formation of a cyclic transition state involving the carbon of the carboxyl group and the reactant is essential for transesterification and hydrolysis reactions. Finally, transesterification under acidic conditions was analyzed in detail in terms of activation energy and ESP obtained from molecular orbital calculations. The reactivity of triglycerides, monoglycerides and diglycerides, and the reaction pathway of center and outside ester bonds are analyzed. It was found that the acid-catalyzed reaction is slower than the base-catalyzed reaction, owing to the formation of a larger, more stable cyclic transition state.

\section{References:}

Alenezi R., Leeke, G.A., Santos R.C.D., Khan A.R. (2009), Hydrolysis kinetics of sunflower oil under subcritical water conditions. Chemical Engineering Research and Design, 87, 867-873.

Asakuma Y., Maeda K., Kuramochi H., Fukui, K. (2009). Theoretical study of the transesterification of triglycerides to biodiesel fuel. Fuel, 88, 786-791.

Barakos N., Pasias S. and Papayannakos N. (2008). Transesterification of triglycerides in high and low quality oil feeds over an HT2 hydrotalcite catalyst. Bioresource Technology, 99, 5037-5042.

Boocock D.G.B., Konar S.K., Mao V., Lee C., Buligan S (1998). Fast Formation of High-Purity Methyl Esters from Vegetable Oils. JAOCS, 75, 1167-1172.

Demirbas A. (2007). Progress and recent trends in biofuels. Progress in Energy Combustion Science, 33, 1-18.

Demirbas A. (2008). Comparison of transesterification methods for production of biodiesel from vegetable oils and fats. Energy Conversion Management, 49, 125-130.

Hori K., Hashitani Y., Kaku Y. and Ohkubo K. (1999). The role of water for the exchange reaction. Journal of Molecular Structure THEOCHEM, 461-462, 589-596.

Jin F. et al. (2007) NMR spectroscopic study on methanolysis reaction of vegetable oil. Fuel, 86, 12011207.

Neyda C. et al. (2008). Transesterification of Jatropha curcas oil glycerides, Theoretical and experimental studies of biodiesel reaction. Fuel, 87, 2286-2295.

Maeda K. et al. (2008). Phase equilibrium of biodiesel compounds for the triolein-palmitic acid-methanol system with dimethyl ether as cosolvent. Chemical Engineering data, 53, 973-977.

Minami E., Saka S. (2006). Kinetics of hydrolysis and methyl esterification for biodiesel production in two-step supercri tical methanol process. Fuel, 85, 2479-2483.

Sharma, Y.C., Singh, B., Upadhyay S.N. (2008). Advancements in development and characterization of biodiesel: A review. Fuel, 87, 2355-2373. 